\title{
The Outcomes of Cemented Femoral Revisions for Periprosthetic Femoral Fractures in the Elderly: Comparison with Cementless Stems
}

\author{
Pavel Sponer (iD ${ }^{1,2}$ \\ Martin Korbel ${ }^{1,2}$ \\ Michal Grinac ${ }^{1,2}$ \\ Libor Prokes ${ }^{1,2}$ \\ Ales Bezrouk (iD ${ }^{3}$ \\ Tomas Kucera (iD) ${ }^{1,2}$ \\ 'Department of Orthopedic Surgery, \\ University Hospital in Hradec Králové, \\ Hradec Králové, Czech Republic; \\ ${ }^{2}$ Charles University, Faculty of Medicine \\ in Hradec Králové, Hradec Králové, \\ Czech Republic; ${ }^{3}$ Department of Medical \\ Biophysics, Charles University in Prague, \\ Faculty of Medicine in Hradec Králové, \\ Hradec Králové, Czech Republic
}

\begin{abstract}
Introduction: The present study compares the outcome of the long cemented stem and the revision uncemented stem used in periprosthetic femoral fractures. We propose that the revision with a long stem cemented prosthesis does not compromise fracture healing.

Patients and Methods: A consecutive series of 37 patients, operated between 2010 and 2017, were enrolled in a retrospective analysis. A long cemented stem was implanted in 21 patients (study group; age at operation: 63 to 89 years). A distally tapered fluted uncemented stem was used in 16 patients (control group; age at operation: 35 to 77 years). The clinical outcome was evaluated with Merle d'Aubigné and Postel scoring system. Standard radiographs were taken before surgery, at 3, 6, and 12 months postoperatively, and last follow-up. Any and all complications during the follow-up period were recorded.

Results: Although a significant difference $(P=0.006)$ was observed in the post-operative Merle d'Aubigné score over the 12-month follow-up period, no significant difference $(P=0.066)$ was found in the post-operative pain score between the study and control groups. Periodic radiographic assessments showed the disappearance of radiolucent lines and the diaphyseal part of the fracture was healed in all 34 followed-up cases during the first annual follow-up. Early surgical complications were seen in both groups, the medical complications were observed only in the study group.

Conclusion: Based on our results, periprosthetic fractures of the femur after a total hip arthroplasty were associated with significant morbidity and increased mortality in elderly patients. Revision with a long-stem cemented prosthesis provided early pain-free weightbearing without compromising the healing of femoral fractures in elderly patients with osteoporotic bone, altered mobility, poor balance, and reduced cognitive capacity.
\end{abstract}

Keywords: revision total hip arthroplasty, periprosthetic hip fractures, cemented stem

\section{Introduction}

The management of periprosthetic fractures of the femur after a total hip arthroplasty can be challenging. The rising incidence of primary total hip arthroplasty, along with an ageing population that is more susceptible to falling trauma, results in a substantial increment of revision operations. ${ }^{1}$ From this perspective, orthopedic surgeons will face a greater number of periprosthetic fractures in elderly patients with osteoporotic bone, altered mobility, poor balance, and reduced cognitive capacity. ${ }^{2}$

Elderly patients with periprosthetic fractures have a higher mortality risk, as well as greater surgical and medical complications. ${ }^{3}$ Therefore, the goals in primary management for elderly patients are vastly different from those observed in younger
Correspondence: Pavel Sponer

Tel +420495833566

Email pavel.sponer@fnhk.cz 
patients. In brief, surgery seeks an early, pain-free, weightbearing status without compromising the healing of femoral fractures. ${ }^{4}$ Revision with a long-stem cemented prosthesis can be used in these cases as it often allows an earlier weight-bearing ability. ${ }^{5}$ However, there are few reports available on the outcome of long cemented stems in elderly patients with periprosthetic fractures. ${ }^{4,6}$

This study evaluates a consecutive series of patients with periprosthetic fractures of the femur after total hip arthroplasty treated with a long cemented stem, focusing on the benefits and risks of surgery. Thus, we compared (1) the hip function, (2) the radiographic outcome of fracture healing, and (3) the complications after revisions performed for periprosthetic fractures with the long stem cemented prosthesis and the distally tapered fluted uncemented stem to determine the efficacy of this technique. Our hypothesis was that revision with a long stem cemented prosthesis does not compromise fracture healing. Further, we theorized that both morbidity and mortality would be significantly pronounced in elderly patients with a long cemented stem.

\section{Patients and Methods}

\section{Patients}

All patients with a periprosthetic fracture of the femur after a total hip arthroplasty, having femoral revision at our institution between January 2010 and December 2017, were enrolled in this retrospective analysis (Supplementary Table 1). In total, 37 revision hip arthroplasties were done in 16 men (43\%) and 21 women (57\%). A long cemented stem was implanted in 15 elderly patients with considerable comorbidity level (ASA score $\geq 3$, Charlson comorbidity index $\geq 4$ ) and 6 patients with expected non-compliance with postoperative non-weight bearing (Figure 1). These 21 patients, with a mean age at operation 79 years (63 to 89), comprised the study group. Uncemented femoral revision was performed in 16 patients without significant comorbidity and that were deemed compliant with protected weight bearing after surgery; these patients constitute the control group, mean age at operation 63 years (35 to 77) (Table 1). The Ethics Committee of the University Hospital in Hradec Kralove approved the study protocol (reference number 202103P06) and waived the requirement for informed consent due to the retrospective design. The accessed patient data complied with relevant protection, privacy guidelines and regulations. The study was performed in accordance with the ethical standards described in the Helsinki Declaration.

\section{Surgical Procedure}

All the procedures were performed in one specialized arthroplasty medical center by four experienced orthopedic surgeons using a direct lateral approach in supine position of the patient. First, the hip joint was opened and dislocated; the cup was assessed for fixation stability and the polyethylene liner for wear. The lateral approach was extended distally; the fracture site was then used for removal of the stem and cement. After additional reaming of the diaphysis, the trial stem was inserted in the medullary canal bypassing the distal fracture site by at least 2 diaphyseal diameters to achieve sufficient stability. The fracture fragments were reduced and held with clamps, followed by trial reduction. After evaluation of the extremity length and implant stability, at least 2 cerclage strips were attached to the femur and the long stem was set in place with the correct anteversion. In the study group, the medullary canal was filled with cement in a retrograde manner before stem insertion and the fracture site was double-checked for cement leakage, with the subsequent removal of any extruded material.

Postoperatively, all patients received prophylactic intravenous antibiotics for 24 hours. Venous thromboprophylaxis was done with low-molecular-weight heparin for 5 weeks. Postoperative physiotherapy consisted of physical exercise focused on muscle strengthening and mobilization of the hip. Mobilization was started on the first postoperative day in a high vertical walker. Patients with a cemented long stem were allowed to bear weight as tolerated; on the other hand, protected weight bearing was recommended for up to 12 weeks until evidence of radiological healing occurred after uncemented femoral revision.

\section{Outcome Assessments}

The clinical outcome was evaluated with Charnley's modified Merle d'Aubigné and Postel scoring system. ${ }^{7}$ The pain score component in this method was evaluated separately because the result of the original scoring system includes objective clinical parameters other than pain. The functional status was determined through the independent ability to walk, making a clear distinction between the following two observations:

1. Maintained independent ambulatory ability: able to walk without support from another person (with aid if necessary). 


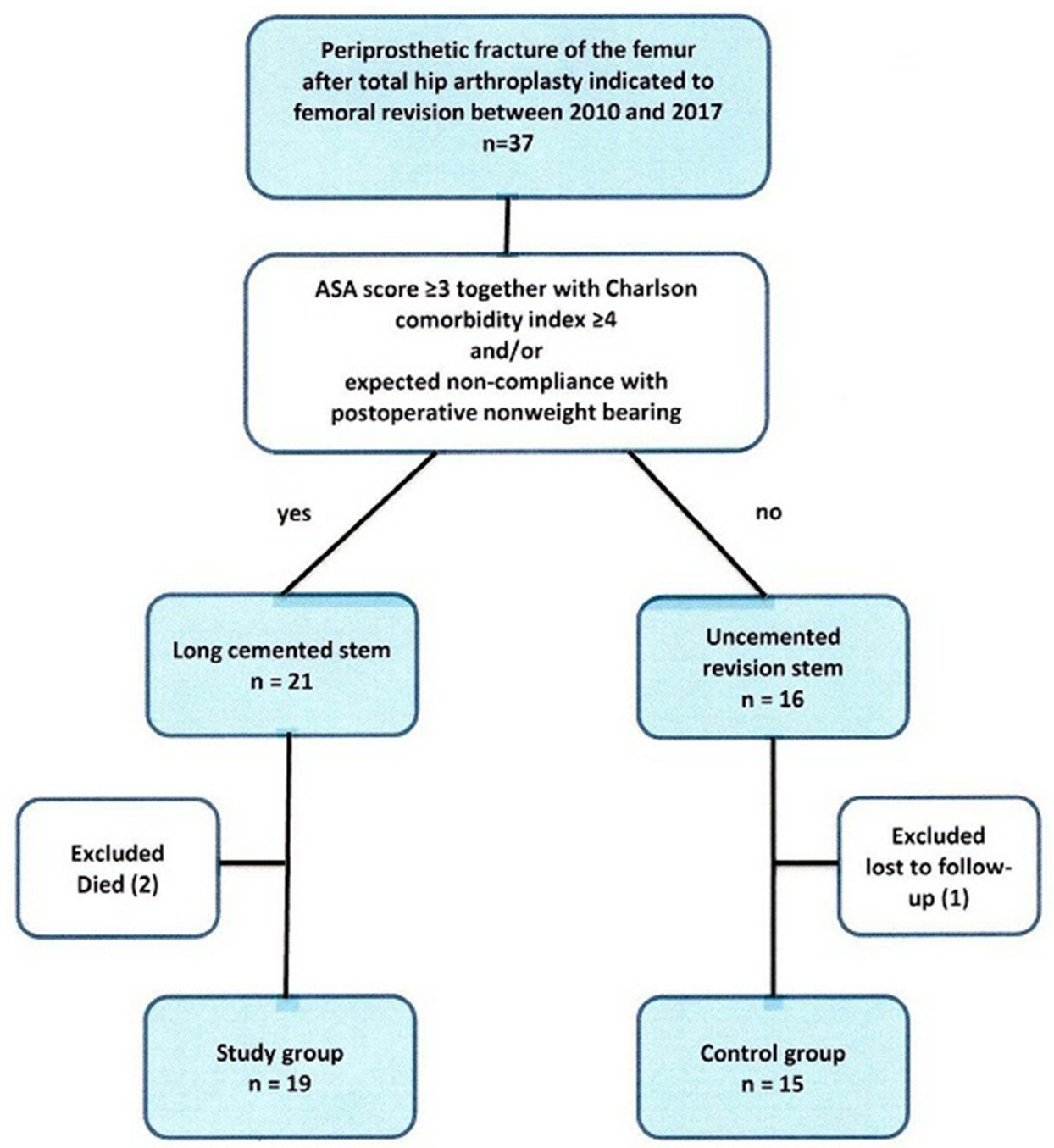

Figure I The study flow-chart.

2. Not maintained: assistance from another person or use of a wheelchair required. ${ }^{8}$

The standard radiographs were evaluated separately by three orthopedists, and the data revised for inter-observer agreement. In case of any observed difference, the patient's radiograph was studied by all three reviewers together. Fracture union was characterized by the presence of bone bridging in both the anteroposterior and lateral radiographs.

Complications were registered throughout the followup period. All patients were under followed-up before surgery, at 3, 6, and 12 months postoperatively, and at the time of the latest appointment. The mean follow-up period was $55(0-99)$ months for the study group $(\mathrm{n}=21)$, whereas that the mean follow-up was 62 (7-124) months for the control group $(\mathrm{n}=16)$.

\section{Statistical Analysis}

The measurement data were processed with the help of MS Excel 2007 (Microsoft Corp, Redmond WA, USA), NCSS 2007 (Hintze, J. (2007). NCSS 2007. NCSS LLC, Kaysville, UT, USA), and PASS 13 Power Analysis and Sample Size Software (Hintze, J. (2014). NCSS, LLC. Kaysville, UT, USA). With respect to the structure of the tested data, we opted for a Wilcoxon Signed-Rank Test (Paired Difference) or a Mann-Whitney $U$-test. The parameters with low number of observations in the tested groups were evaluated with a Freeman-Halton modification (Freeman and Halton, 1951) of Fischer's exact test. In all the tests, a value of $P<0.05$ was considered as significant. 
Table I Demographics of Patients

\begin{tabular}{|l|c|c|}
\hline & $\begin{array}{c}\text { Study } \\
\text { Group }\end{array}$ & $\begin{array}{c}\text { Control } \\
\text { Group }\end{array}$ \\
\hline Number of patients & 21 & 16 \\
\hline Number of hips & 21 & 16 \\
\hline Age (mean; range; years) & $79(63-89)$ & $63(35-77)$ \\
\hline $\begin{array}{l}\text { Gender } \\
\text { Female }\end{array}$ & 14 & 7 \\
Male & 7 & 9 \\
\hline Right side & 14 & 9 \\
\hline Left side & 7 & 7 \\
\hline ASA score, average & 2.81 & 2.25 \\
ASA-I & - & 1 \\
ASA-2 & 6 & 11 \\
ASA-3 & 13 & 3 \\
ASA-4 & 2 & 1 \\
\hline Charlson comorbidity index, average & 4.76 & 2.31 \\
\hline $\begin{array}{l}\text { Time from admission to surgery (mean; } \\
\text { range; days) }\end{array}$ & $5.2(1-13)$ & $4.9(2-8)$ \\
\hline
\end{tabular}

\section{Results}

\section{Study Design and Patients}

The scheduled clinical and radiographic follow-up was accomplished in 34 patients within the first year after surgery. Two patients in the study group died within the first month after surgery; one patient in the control group was lost to follow-up after 7 months.

Of the 19 remaining patients in the study group, eight had died at time of review, whereas that of the remaining 15 patients in the control group, one patient was deceased from causes unrelated to the surgery (pulmonary tumor).

\section{Clinical Results}

Whereas no significant difference was found in the postoperative pain score $(P=0.066)$, a significant difference in the post-operative Merle d'Aubigné score was observed between the study and control groups $(P=0.006)$ over the 12-month follow-up period (Table 2).

The functional status of the patients in the study group deteriorated over time. Further, all but one of the patients were able to walk independently before surgery; in contrast, at the time of the latest follow-up, 8 out of 11 living patients (73\%) were still able to walk independently with the aid of forearm crutches. All followed-up patients in the control group maintained independent ambulatory capacity at the time of review.

\section{Radiography}

The radiographs obtained immediately after surgery in all 37 patients proved the correct position of the revision prosthesis and adequate stability of the femoral fracture. Periodic assessments showed that, during the 3-12 months period, the radiolucent lines disappeared and the fractures were united at the first annual follow-up in all but one of the 34 followed-up cases (Figures 2 and 3). One patient in the study group had a pain-free non-union of the greater trochanter, showing a healed diaphyseal section of the fracture during the final follow-up, 5 years after the surgery.

\section{Complications}

The observed complications are shown in Table 3. Whereas early surgical complications were seen in both groups, the medical complications were observed only in the study group.

Dislocations happened in two patients within the first months postoperatively in the study group. One patient underwent an efficient closed reduction of a correctly implanted prosthesis and one patient had a successful conversion to a constrained liner for recurrent instability. Two patients in the control group had a deep infection; however, they were successfully treated with surgical debridement and the mobile parts were exchanged, this was followed by 6 weeks of antibiotic therapy. One patient in the control group suffered from prolonged unrecovered peroneal nerve palsy, which was managed conservatively. Following surgery, 6 patients in the study group $(28.5 \%)$ suffered medical complications, the most common of which was pneumonia $(n=3)$.

Two patients of the study group and one patient of the control group had late hip-related complications. In the study group, one late deep infection occurred 30 months after revision surgery and antimicrobial suppression was chosen because the existing comorbidities did not allow additional surgery. In one patient of the study group, a cemented stem had been removed because of recurrent dislocation developed after a fatal stroke 6 years postoperatively. One patient in the control group had late deep infection; however, she was successfully treated with two-stage exchange arthroplasty.

Two patients in the study group died - both of pneumonia, within one month postoperatively. The overall 1-year mortality in patients with periprosthetic fractures of the femur treated with the long cemented 
Table 2 Clinical Results of Patients

\begin{tabular}{|c|c|c|}
\hline & Study Group ${ }^{+}$ & Control Group ${ }^{++}$ \\
\hline Follow-up (mean; range; months) & $55(0-99)$ & $62(7-124)$ \\
\hline Preoper.pain score \pm the postoper.increase at 12 months postoperatively (mean; points) & $1+4.8$ & $1+4.7$ \\
\hline Hip flexion at 12 months postoperatively (mean; range; degrees) & $86(60-120)$ & $88(70-100)$ \\
\hline Hip abduction at 12 months postoperatively (mean; range; degrees) & $29(15-40)$ & $29(15-40)$ \\
\hline $\begin{array}{l}\text { Preoper.Merle d'Aubigné and Postel score } \pm \text { the postoper.increase at } 12 \text { months } \\
\text { postoperatively (mean; points) }\end{array}$ & $2+10.7$ & $2+12.5$ \\
\hline \multicolumn{3}{|l|}{ Functional status preoperatively } \\
\hline Independent ability to walk & 20 & 16 \\
\hline Support from another person or use of wheelchair & I & - \\
\hline \multicolumn{3}{|l|}{ Functional status at the time of latest follow-up } \\
\hline Independent ability to walk & 14 & 16 \\
\hline Support from another person or use of wheelchair & 7 & - \\
\hline
\end{tabular}

Notes: ${ }^{+}$Two patients lost to further follow-up within the first month after surgery. ${ }^{++}$One patient lost to further follow-up 7 months after surgery.

stem was estimated in $9.5 \%$. Eight patients in the study group and one patient in the control group were deceased at the time of review from causes unrelated to the surgery.

\section{Discussion}

Although most authors advise revision with a long stem prosthesis used as an intramedullary rod in the treatment

A

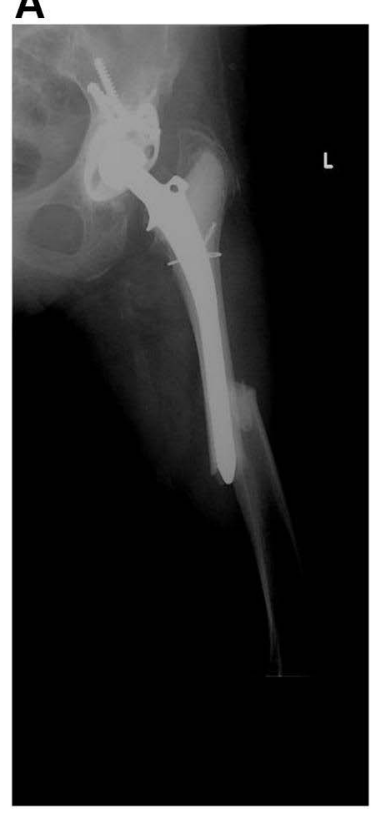

B

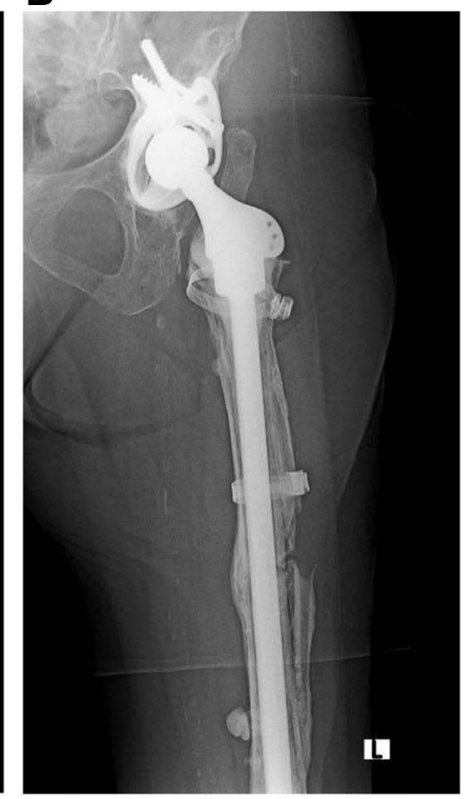

Figure 2 Preoperative anteroposterior radiograph of a Vancouver type BI periprosthetic femoral fracture in a 77-year-old female $(\mathbf{A})$ treated with a long cemented femoral stem (B). At 5 years of follow-up, the fracture was healed and the stem did not show any sign of aseptic loosening. of Vancouver B2 and B3 periprosthetic femoral fractures, there is a continuous debate on whether cemented or uncemented implants should be used. ${ }^{9-14}$ Our study demonstrates a clear improvement in hip/thigh pain relief following revision total hip arthroplasty in patients with periprosthetic fractures. We suppose that the significant difference in post-operative Merle d'Aubigné and Postel scores found between the study and control groups was
A

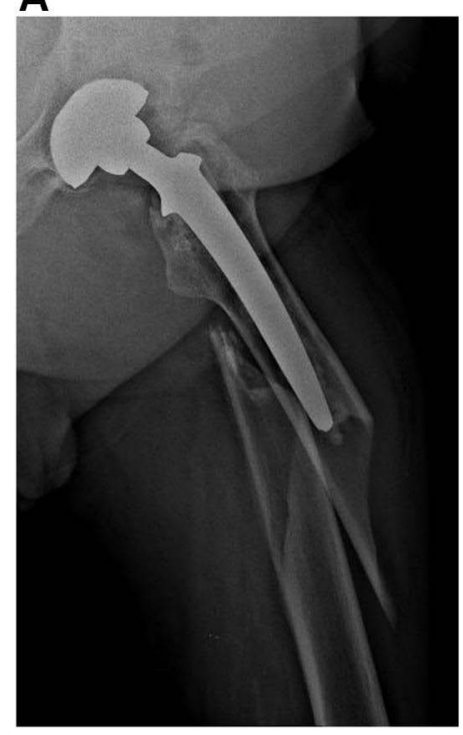

B

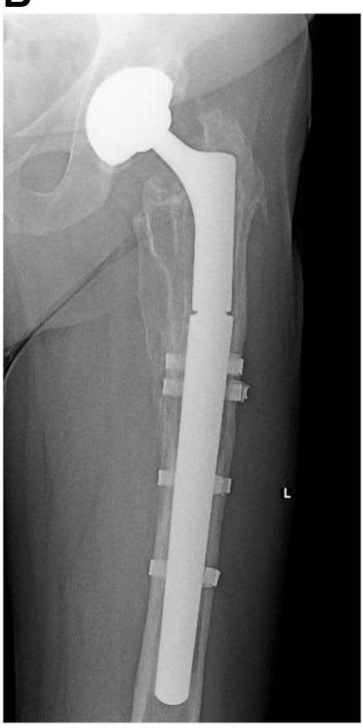

Figure 3 Preoperative anteroposterior radiograph of a Vancouver type-B2 periprosthetic femoral fracture in a 61 -year-old man (A) treated with an uncemented femoral revision (B). Fracture was healed and uncemented modular tapered fluted revision stem was well osteointegrated after I year of surgery. 
Table 3 Early and Late Complications

\begin{tabular}{|c|c|c|}
\hline \multirow[t]{2}{*}{ Complications } & Study Group & Control Group \\
\hline & $\begin{array}{c}\text { (2 I Hips; } 2 \text { I } \\
\text { Patients) }\end{array}$ & $\begin{array}{c}\text { (16 Hips; } 16 \\
\text { Patients) }\end{array}$ \\
\hline Early (< 3 months) & 17 & 4 \\
\hline Dislocation & 2 & - \\
\hline Periprosthetic infection & - & 2 \\
\hline Peroneal nerve palsy & - & 1 \\
\hline Pneumonia & 3 & - \\
\hline Pressure sores & 3 & I \\
\hline Electrolyte imbalance & 2 & - \\
\hline Ventricular tachyfibrillation & 2 & - \\
\hline Postoperative confusion & 2 & - \\
\hline Aspiration & I & - \\
\hline Cerebrovascular accident & I & - \\
\hline Urinary tract infection & 1 & - \\
\hline Late (> 3 months) & 2 & I \\
\hline Dislocation & I & - \\
\hline Periprosthetic infection & I & I \\
\hline TOTAL & 19 & 5 \\
\hline
\end{tabular}

affected by the deteriorated functional status of elderly patients with considerable high comorbidity level in the study group. The radiographic evaluation showed that revision with a long stem cemented prosthesis did not compromise fracture healing because the diaphyseal section of the fracture was found completely healed during the first annual follow-up meeting in the 19 cases evaluated in this period. Although early surgical complications were recorded in both groups, morbidity and increased medical complications were predominant in our study group.

However, this case-control study is limited by the small number of patients and short follow-up duration. Regardless, all patients with a periprosthetic fracture of the femur after a total hip arthroplasty having femoral revision at our tertiary referral hospital during an 8 years period were enrolled in a retrospective survey. Regrettably, the available literature is rather lacking in long-term follow-up of larger series comparing long stem cemented prosthesis and distally tapered fluted uncemented stem in periprosthetic femoral fractures. Nevertheless, the followup period in our analysis was sufficient to reveal the healing of fractures. Furthermore, the number of patients and follow-up period is comparable to other published surveys on long cemented stem in patients with periprosthetic fractures. ${ }^{4,6} \mathrm{We}$ are aware of the significant differences in baseline characteristics between the groups. Fractures in the control group with the revision uncemented implant occurred at a younger age than the study group with a long cemented stem. The shorter follow-up of the cemented group reflects the frailty of the elderly patients, who were significantly older, in comparison with those in the uncemented group.

Several disadvantages have been previously reported concerning the use of long cemented stems. ${ }^{6}$ Cement may be extruded into the fracture site, which could be the reason of non-union or refracture. ${ }^{15}$ Whereas Corten et al published a healed diaphyseal section of a fracture in sixteen followed-up patients after more than 1 year, Springer et al reported three (9\%) out of thirty-four unrevised cemented stems with radiographic non-union after 68 months of follow-up. ${ }^{4,6}$ The non-union rate could be diminished with thorough handling of the surrounding soft tissues to prevent bone devascularization; further, careful inspection and removal of excess cement and the use of autogenous bone grafts or cortical strut allografts could also alleviate this issue. ${ }^{6}$ Aseptic loosening is another concern regarding cemented stems. On the one hand, Corten et al demonstrated no revision of sixteen patients due to aseptic loosening; in contrast, Springer et al found twenty-five $(60 \%)$ out of forty-two cemented stems with a well-fixed, unrevised implant and healed periprosthetic fracture. ${ }^{4,6}$ In the present study, we identified seventeen (81\%) out of twenty-one well fixed, unrevised long cemented stems during the last follow-up. Finally, there is an indication for greater early mortality if cement is used. ${ }^{16}$ However, the high perioperative mortality rate seems to be rather patient- and procedure-related rather than implant-related.

In our study, the overall 1-year mortality in elderly patients with periprosthetic fractures treated with the long cemented stem was of $9.5 \%$. In this regard, Young et al reported a six-month overall mortality of $0.9 \%$ after revision total hip arthroplasty compared to $7.3 \%$ after revision surgery for periprosthetic fracture. ${ }^{17}$ After revision hip arthroplasty, Yao et al found a greater six-month, oneyear, and five-year mortality rate for periprosthetic fractures than for loosening/wear and/or infection. ${ }^{18}$ Recently, Gibbs et al found that hospital-acquired pneumonia and dislocation were associated with increased one-year mortality rates after revision hip arthroplasty for periprosthetic fractures. ${ }^{19}$ Compared to this study, our dislocation rate $(10.5 \%)$ in the elderly patients with long cemented stems was similar 
$(10.8 \%)$. We observed pneumonia in two patients deceased within the first month after surgery.

Revision surgery is a major procedure that involves an extended operating time, substantial blood loss, and considerable physiological stress. ${ }^{20,21}$ Revision hip arthroplasty for periprosthetic femoral fracture differs from other common indications, where the elective procedure is performed after preoperative optimization of the patient. ${ }^{22,23}$ Based on recent studies, surgery time was not identified as a risk factor for mortality in periprosthetic fractures. ${ }^{19,24}$ This suggests that the selection of appropriate surgical and anesthetic options may be crucial for the preoperative optimization of the patient. For elderly patients with poor balance, postoperative partial weight bearing after hip revision might be more difficult. $^{25}$ Therefore, the use of long-stem cemented prosthesis could allow early weight bearing in elderly and morbid patients or in patients non-compliant with partial weight bearing after hip revision.

\section{Conclusions}

Based on the obtained results in our study, the periprosthetic fractures of the femur after a total hip arthroplasty are associated with significant morbidity and increased mortality in the elderly. Revision with a long-stem cemented prosthesis provides early pain-free weight-bearing without compromising the healing of the femoral fracture in elderly patients with osteoporotic bones, altered mobility, poor balance, and reduced cognitive capacity. However, the management of periprosthetic fractures around the femoral stem requires extensive surgical expertise.

\section{Ethical Statement}

Informed consent regarding surgery was obtained from all patients before intervention. The Ethics Committee of the University Hospital in Hradec Kralove approved the study protocol (reference number 202103P06) and waived the requirement for informed consent due to the retrospective design. The patient data accessed complied with relevant patient data protection and privacy regulations. The study was performed in accordance with the ethical standards of the Helsinki Declaration.

\section{Funding}

This work was made possible thanks to the grant PROGRES Q40/04 and MHCZ - DRO (UHHK, 00179906).

\section{Disclosure}

Prof. Dr. Pavel Sponer reports grants from Charles University in Prague and from Ministry of Health of the Czech Republic, Dr. Tomas Kucera and Dr. Michal Grinac report grant from Charles University in Prague, during the conduct of the study. The authors have no conflict of interest to disclose in relation to this article.

\section{References}

1. Kurtz S, Ong K, Lau E, Mowat F, Halpern M. Projections of primary and revision hip and knee arthroplasty in the United States from 2005 to 2030. J Bone Joint Surg Am. 2007;89:780-785. doi:10.2106/ 00004623-200704000-00012

2. Korbel M, Sponer P, Kucera T, Procházka E, Procek T. Results of treatment of periprosthetic femoral fractures after total hip arthroplasty. Acta Med. 2013;56:67-72. doi:10.14712/ 18059694.2014.26

3. Märdian S, Perka C, Schaser KD, Gruner J, Scheel F, Schwabe P. Cardiac disease and advanced age increase the mortality risk following surgery for periprosthetic femoral fractures. Bone Joint J. 2017;99B:921-926. doi:10.1302/0301-620X.99B7.BJJ-2016-0974.R1

4. Corten K, Macdonald SJ, McCalden RW, Bourne RB, Naudie DD. Results of cemented femoral revisions for periprosthetic femoral fractures in the elderly. $J$ Arthroplasty. 2012;27:220-225. doi:10.1016/j.arth.2011.03.050

5. Haasper C, Enayatollahi MA, Gehrke T. Treatment of Vancouver type B2 periprosthetic femoral fractures. Int Orthop. 2015;39:1989-1993. doi:10.1007/s00264-015-2959-4

6. Springer BD, Berry DJ, Lewallen DG. Treatment of periprosthetic femoral fractures following total hip arthroplasty with femoral component revision. J Bone Joint Surg Am. 2003;85-A:2156-2162. doi:10.2106/00004623-200311000-00015

7. Charnley J. The long-term results of low-friction arthroplasty of the hip performed as a primary intervention. J Bone Joint Surg Br. 1972;54:61-76. doi:10.1302/0301-620X.54B1.61

8. Pedersen TJ, Lauritsen JM. Routine functional assessment for hip fracture patients: are there sufficient predictive properties for subgroup identification in treatment and rehabilitation? Acta Orthop. 2016;87:374-379. doi:10.1080/17453674.2016.1197534

9. Duncan CP, Masri BA. Fractures of the femur after hip replacement. Instr Course Lect. 1995;44:293-304.

10. Tsiridis E, Pavlou G, Venkatesh R, Bobak P, Gie G. Periprosthetic femoral fractures around hip arthroplasty: current concepts in their management. Hip Int. 2009;19:75-86. doi:10.1177/1120700009 01900201

11. Duncan CP, Haddad FS. The Unified Classification System (UCS): improving our understanding of periprosthetic fractures. Bone Joint J. 2014;96-B:713-716. doi:10.1302/0301-620X.96B6.34040

12. Munro JT, Garbuz DS, Masri BA, Duncan CP. Tapered fluted titanium stems in the management of Vancouver B2 and B3 periprosthetic femoral fractures. Clin Orthop Relat Res. 2014;472:590-598. doi:10.1007/s11999-013-3087-3

13. Khan T, Grindlay D, Ollivere BJ, Scammell BE, Manktelow AR, Pearson RG. A systematic review of Vancouver B2 and B3 periprosthetic femoral fractures. Bone Joint J. 2017;99-B:17-25. doi:10.1302/ 0301-620X.99B4.BJJ-2016-1311.R1

14. Hevesi M, Wyles CC, Yao JJ, et al. Revision total hip arthroplasty for the treatment of fracture: more expensive, more complications, same diagnosis-related groups: a local and national cohort study. $J$ Bone Joint Surg Am. 2019;101:912-919. doi:10.2106/JBJS.18.00523 
15. Crockarell JR, Berry DJ, Lewallen DG. Nonunion after periprosthetic femoral fracture associated with total hip arthroplasty. J Bone Joint Surg Am. 1999;81:1073-1079. doi:10.2106/00004623-19990800000003

16. Garland A, Gordon M, Garellick G, Kärrholm J, Sköldenberg O, Hailer NP. Risk of early mortality after cemented compared with cementless total hip arthroplasty: a nationwide matched cohort study. Bone Joint J. 2017;99-B:37-43. doi:10.1302/0301620X.99B1.BJJ-2016-0304.R1

17. Young SW, Walker CG, Pitto RP. Functional outcome of femoral periprosthetic fracture and revision hip arthroplasty: a matched-pair study from the New Zealand Registry. Acta Orthop. 2008;79:483-488. doi:10.1080/17453670710015463

18. Yao JJ, Maradit Kremers H, Abdel MP, et al. Long-term mortality after revision THA. Clin Orthop Relat Res. 2018;476(2):420-426. doi: $10.1007 / \mathrm{s} 11999.0000000000000030$

19. Gibbs VN, McCulloch RA, Dhiman P, et al. Modifiable risk factors for mortality in revision total hip arthroplasty for periprosthetic fracture. Bone Joint J. 2020;102-B:580-585. doi:10.1302/0301620X.102B5.BJJ-2019-1673.R1

20. Köksal A, Öner A, Çimen O, et al. Femoral stem fractures after primary and revision hip replacements: a single-center experience. J Dis Relat Surg. 2020;31(3):557-563.
21. Çağlar Ö, Tokgözoğlu M, Akgün RC, Atilla B. Low-dose vancomycin-loaded cement spacer for two-stage revision of infected total hip arthroplasty. J Dis Relat Surg. 2020;31(3):449-455.

22. Khan T, Middleton R, Alvand A, Manktelow ARJ, Scammell BE, Ollivere BJ. High mortality following revision hip arthroplasty for periprosthetic femoral fracture. Bone Joint J. 2020;102B:1670-1674. doi:10.1302/0301-620X.102B12.BJJ-2020-0367.R1

23. Ramavath A, Lamb JN, Palan J, Pandit HG, Jain S. Postoperative periprosthetic femoral fracture around total hip replacements: current concepts and clinical outcomes. EFORT Open Rev. 2020;5 (9):558-567. doi:10.1302/2058-5241.5.200003

24. Boronratwet $\mathrm{P}, \mathrm{Fu} \mathrm{MC}$, Adrados M, et al. Unlike native hip fractures, delay to periprosthetic hip fracture stabilization does not significantly affect most short-term perioperative outcomes. $J$ Arthroplasty. 2019;34:564-569. doi:10.1016/j.arth.2018.11.006

25. Mulay S, Hassan T, Birtwistle S, Power R. Management of types B2 and B3 femoral periprosthetic fractures by a tapered, fluted, and distally fixed stem. J Arthroplasty. 2005;20:751-756. doi:10.1016/j. arth.2004.11.020
Clinical Interventions in Aging

\section{Publish your work in this journal}

Clinical Interventions in Aging is an international, peer-reviewed journal focusing on evidence-based reports on the value or lack thereof of treatments intended to prevent or delay the onset of maladaptive correlates of aging in human beings. This journal is indexed on PubMed Central, MedLine, CAS, Scopus and the Elsevier

\section{Dovepress}

Bibliographic databases. The manuscript management system is completely online and includes a very quick and fair peer-review system, which is all easy to use. Visit http://www.dovepress.com/ testimonials.php to read real quotes from published authors. 